\title{
Office Laboratory Regulations
}

The number and scope of laboratory tests which can be done in the clinician's office have dramatically increased in the past ten years. This testing has been made possible by new technology and has been promoted by a shift in health care from the inpatient to the outpatient environment. With these changes has come a growing concern about the quality of the testing performed in office laboratories. This concern led to several articles in the press, and, eventually, the attention of Congress. In late 1988, the Clinical Laboratory Improvement Amendment (P.L. $100-578$ or CLIA 88) was passed. Under this law, all office laboratories are required to be licensed by January 1, 1990. (Prior to CLIA 88, a Medicare amendment had been passed which called for regulation of laboratories performing more than 5,000 tests per year. This 5,000 test limit is no longer applicable.)

Under the new bill, laboratories which use only very simple tests will be licensed but will be waivered from full regulation. The Health Care Financing Administration (HCFA) is now in the process of developing guidelines to define such waivered laboratories. These laboratories will apply to HCFA for a license and will be charged a small registration fee. It is likely that a majority of office laboratories will be unwaiverable and will therefore come under more extensive regulations.

The CLIA 88 bill specifies that HCFA can give "deemed status" to voluntary, nonprofit organizations to accredit laboratories. The American Academy of Family Physicians has joined with the College of American Pathologists, the American Society of Internal Medicine, and the American Medical Association to form the Commission on Office Laboratory Assessment: COLA is the process of securing deemed status from HCFA. To date, no other medical association has developed a similar office laboratory accreditation program. COLA accreditation applictions are now available (8701 Georgia Avenue, Suite 403-B, Silver Spring, Maryland 20910, (301) 588-5882).

There are four elements for COLA accreditation. The first is a personnel requirement. It is very clear from the CLIA 88 bill that of fice laboratories are not required to hire formally trained technicians or technologists. However, the clinician must be sure that the people who perform the tests are properly trained. In the COLA accreditation package, a per- sonnel form is filled out for each person in the office who performs any testing. The form lists what tests are done and how the office staff were trained (i.e., self trained, trained by the physician, formal training, trained by a salesperson).

The second element is "quality assurance". Hospital and reference laboratories spend about $30 \%$ of their total laboratory budgets on the quality controls that are done to ensure that the tests are accurate. The COLA accreditation application reviews the quality assurance in your laboratory with a "checklist". This checklist covers 170 different quality control items that are considered to be "good laboratory practice" (i.e., is the chemistry instrument calibrated at appropriate intervals?).

COLA accreditation rests on a laboratory's successful performance in a proficiency testing program. These programs send out "unknown" specimens to your laboratory every three months. You then test the specimen, send in an answer sheet, and are graded in comparison to other participants. COLA approved proficiency testing progrms are now available from the College of American Pathologists, American Society of Internal Medicine, and the American Association of Bioanalysts. The AAFP is developing a proficiency testing program designed for family physicians. This program should be available by January 1, 1990 .

The final element of the accreditation process is laboratory inspection. All hospital and reference laboratories are currently inspected every two years. COLA plans to inspect only those laboratories which have problems in their proficiency testing. (CLIA 88 regulations for inspection do not go into effect until June 1991.)

Some of these programs may appear to be an unnecessary burden on physicians. Organized medicine was actively involved in the Washington discussions and the consensus has been that things could have come out much worse. By waiving simple testing, permitting accreditation through voluntary organizations (rather than directly through HCFA) and removing the need to hire trained technologists, most office laboratories will be able to continue to provide rapid test results to physicians and their patients.

Paul M. Fischer, M.D.

Medical College of Georgia

Department of Family Medicine

Augusta, Georgia 30912-3500 